\title{
Simultaneous Qualitation and Quantification of Thirteen Bioactive Compounds in Flos Lonicerae by High-Performance Liquid Chromatography with Diode Array Detector and Mass Spectrometry
}

\author{
Zheng-Ming QIan, ${ }^{a}$ Hui-Jun LI, ${ }^{*, b}$ Ping LI, ${ }^{*, a, b}$ Mei-Ting Ren, ${ }^{a}$ and Dan TAng ${ }^{b}$ \\ ${ }^{a}$ Key Laboratory of Modern Chinese Medicines (China Pharmaceutical University), Ministry of Education; Nanjing, \\ 210009, People's Republic of China: and ${ }^{b}$ Department of Pharmacognosy, China Pharmaceutical University; Nanjing, \\ 210009, People's Republic of China. $\quad$ Received January 30, 2007; accepted March 17, 2007
}

\begin{abstract}
A high-performance liquid chromatography (HPLC) with diode array detector (DAD) and electrospray ionization mass spectrometry (ESI-MS) was established for the simultaneous determination of thirteen bioactive compounds in Flos Lonicerae. The optimal chromatographic conditions were obtained on a $\mathrm{C}_{18}$ column $(250 \times 4.6 \mathrm{~mm}, 5.0 \mu \mathrm{m})$ with the column temperature at $30^{\circ} \mathrm{C}$. The mobile phase was composed of (A) acetic acid aqueous $(0.4 \%, \mathrm{v} / \mathrm{v})$ and $(\mathrm{B})$ acetonitrile using a gradient elution, the flow rate was $1 \mathrm{ml} / \mathrm{min}$. Detection wavelengths were set at $240 \mathrm{~nm}$ for iridoids (loganin, sweroside, secoxyloganin and centauroside), $330 \mathrm{~nm}$ for phenolic acids (chlorogenic acid, caffeic acid, 4,5-di- $O$-caffeoyl quinic acid and 3,4-di- $O$-caffeoyl quinic acid) and $360 \mathrm{~nm}$ for flavonoids (rutin, hyperoside, quercetin-3-O-glucoside, luteolin-7-O-glucoside and lonicerin). The identities of the peaks were accomplished by comparing retention times, UV and mass data with reference compounds under the same conditions. All calibration curves showed good linear regression $\left(r^{2}>0.9983\right)$ within test ranges. The developed method provided satisfactory precision and accuracy with overall intra-day and inter-day variations of $0.78-1.85 \%$ and $1.13-2.36 \%$, respectively, and the overall recoveries of $91.3-104.2 \%$ for the thirteen compounds analyzed. The verified method was successfully applied to quantitative determination of the three types of bioactive compounds in ten commercial Flos Lonicerae samples from different markets in China. The analytical results demonstrated that the contents of the thirteen analytes were relatively variant.
\end{abstract}

Key words HPLC-DAD-MS; Flos Lonicerae; iridoid; phenolic acid; flavonoid

Flos Lonicerae, derived from the dried flower buds of Lonicera japonica THUNB., is one of the most widely used traditional Chinese medicines (TCMs) and recorded in China Pharmacopeia with the Chinese name Jinyinhua for the treatment of acute fever, headache, pharyngodynia, respiratory infection, pyocutaneous disease and epidemic disease. ${ }^{1-3)}$ Pharmacological studies and clinical practice have demonstrated that Flos Lonicerae has many biological functions, including hepatoprotective, cytoprotective, antimicrobial, antibiotic, antioxidative, antiviral and anti-inflammatory activities. $^{4-10)}$ Chemical investigations on Flos Lonicerae resulted in discovering three main types of bioactive compounds, i.e. phenolic acids, iridoids, flavonoids. ${ }^{11)}$

Generally, chlorogenic acid and flavonoids were believed to be the beneficial components and were chosen as marker compounds for the quality evaluation and standardization of Flos Lonicerae and its preparations. ${ }^{12,13)}$ Recently, as another type of pharmacologically important constituents, major iridoids in Flos Lonicerae were determined by two different HPLC methods. ${ }^{14-18)}$ However, due to multiple compounds that might be associated with the therapeutic functions, a single or a few marker compounds could not be responsible for the overall pharmacological acitivities of Flos Lonicerae. Therefore, it is urgently needed to establish a comprehensive quality evaluation method based on analysis of the whole bioactive compounds in order to accurately reflect the quality of this herbal drug.

In this study, the method of HPLC-DAD has been developed for the simultaneously qualitative and quantitative analysis of thirteen bioactive compounds: chlorogenic acid (1), caffeic acid (2), loganin (3), sweroside (4), secoxyloganin (5), rutin (6), hyperoside (7), quercetin-3-O-glucoside (8), luteolin-7-O-glucoside (9), lonicerin (10), 4,5-di-O-caf- feoyl quinic acid (11), 3,4-di- $O$-caffeoyl quinic acid (12) and centauroside (13) in Flos Lonicerae.

\section{Experimental}

Reagents, Chemicals and Samples Acetonitrile was of HPLC grade (Merck, Darmstadt, Germany). Ultra pure water was obtained in a Milli-Q system from Millipore (Bedford, MA, U.S.A.). The other solvents, purchased from Nanjing Chemical Factory (Nanjing, China), were of analytical grade

Thirteen reference compounds were isolated and purified from L. japonica ThunB. by a series of chromatography procedures in our laboratory, and their structures were elucidated by comparison of spectral data (UV, IR, MS

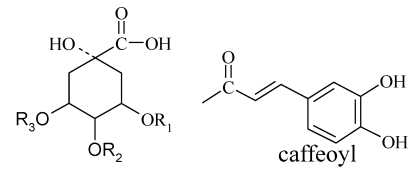

chlorogenic acid (1): $\mathrm{R}_{1}=$ caffeoyl, $\mathrm{R}_{2}=\mathrm{H}, \mathrm{R}_{3}=\mathrm{H}$

4,5- di-O-caffeoyl quinic acid (11) : $\mathrm{R}_{1}=$ caffeoyl, $\mathrm{R}_{2}=$ caffeoyl, $\mathrm{R}_{3}=\mathrm{H}$ 3,4- di-O-caffeoyl quinic acid (12): $\mathrm{R}_{1}=\mathrm{H}, \mathrm{R}_{2}=$ caffeoyl, $\mathrm{R}_{3}=$ caffeoyl
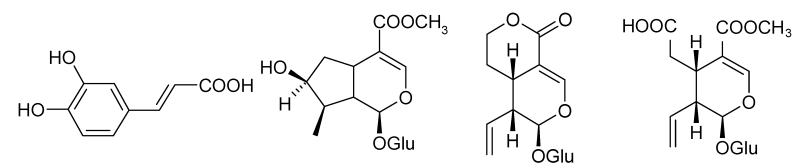

caffeic acid (2) $\quad$ loganin (3)

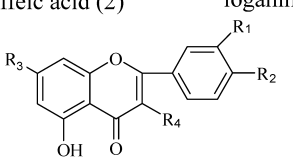

rutin (6): $\mathrm{R}_{1}=\mathrm{OH}, \mathrm{R}_{2}=\mathrm{OH}, \mathrm{R}_{3}=\mathrm{OH}, \mathrm{R}_{4}=\mathrm{Glu}(6-1) \mathrm{Rha}$ hyperoside (7): $\mathrm{R}_{1}=\mathrm{OH}, \mathrm{R}_{2}=\mathrm{OH}, \mathrm{R}_{3}=\mathrm{OH}, \mathrm{R}_{4}=\mathrm{Gal}$ quercetin-3-O-glucoside (8): $\mathrm{R}_{1}=\mathrm{OH}, \mathrm{R}_{2}=\mathrm{OH}, \mathrm{R}_{3}=\mathrm{OH}, \mathrm{R}_{4}=\mathrm{Glu}$ luteolin-7-O-glucoside (9): $\mathrm{R}_{1}=\mathrm{OH}, \mathrm{R}_{2}=\mathrm{OH}, \mathrm{R}_{3}=\mathrm{Glu}, \mathrm{R}_{4}=\mathrm{H}$ lonicerin (10): $\mathrm{R}_{1}=\mathrm{OH}, \mathrm{R}_{2}=\mathrm{OH}, \mathrm{R}_{3}=\mathrm{Rha}(2-1) \mathrm{Glu}, \mathrm{R}_{4}=\mathrm{H}$

Fig. 1. Chemical Structures of Thirteen Compounds in Flos Lonicerae 
$\left.{ }^{1} \mathrm{H}-\mathrm{NMR},{ }^{13} \mathrm{C}-\mathrm{NMR}\right)$ with the literature data: chlorogenic acid (1) ${ }^{19)}$ caffeic acid (2), ${ }^{14)}$ loganin $(\mathbf{3}),{ }^{14)}$ sweroside $(\mathbf{4}),{ }^{14)}$ secoxyloganin $(\mathbf{5}),{ }^{14)}$ rutin $(\mathbf{6}),{ }^{12,20)}$ hyperoside (7), ${ }^{21)}$ quercetin-3-O-glucoside $(\mathbf{8}),{ }^{21)}$ luteolin-7-O-glucoside $(\mathbf{9}),{ }^{12,20)}$ lonicerin $(\mathbf{1 0}),{ }^{19)} 4,5$-di- $O$-caffeoyl quinic acid (11) ${ }^{22)} 3,4$-di- $O$-caffeoyl quinic acid $(\mathbf{1 2})^{22)}$ and centauroside $(\mathbf{1 3})^{23)}$ (Fig. 1). The purity of these reference compounds were determined to be more than $98 \%$ by normalization of the peak areas detected by HPLC analysis.

Ten commercial samples of Flos Lonicerae were purchased at local drug stores from different provinces of China, including Zhenzhou, Henan Province (No. 1), Beijing (No. 2), Taiyuan, Shanxi Province (No. 3), Nanjing, Jiangsu Province (No. 4), Tangshan, Hebei Province (No. 5), Shanghai (No. 6), Chengdu, Sichuan Province (No. 7), Hefei, Anhui Province (No. 8), Jinan, Shandong Province (No. 9), Hangzhou, Zhejiang Province (No. 10). All the samples were authenticated by Professor Ping Li (Department of Pharmacognosy, China Pharmaceutical University), voucher specimens were deposited in the Herbarium of Pharmacognosy, China Pharmaceutical University, Nanjing, China.

HPLC System and Conditions The analysis were performed on an $\mathrm{Ag}$ ilent 1100 liquid chromatography system, equipped with a dual pump, an autosampler and a diode array detector. A Zorbax Extend-C18 column $(250 \times 4.6 \mathrm{~mm}, 5 \mu \mathrm{m})$ at temperature of $30^{\circ} \mathrm{C}$ was applied for all analyses. Detection wavelengths were set at $240 \mathrm{~nm}$ for iridoids (loganin, sweroside, secoxyloganin and centauroside), $330 \mathrm{~nm}$ for phenolic acids (chlorogenic acid, caffeic acid, 4,5-di- $O$-caffeoyl quinic acid and 3,4-di- $O$-caffeoyl quinic acid) and $360 \mathrm{~nm}$ for flavonoids (rutin, hyperoside, quercetin-3- $O$-glucoside, luteolin-7-O-glucoside and lonicerin). The mobile phase consisted of $0.4 \%$ acetic acid aqueous (A) and acetonitrile (B) using a gradient elution of $11 \%$ $\mathrm{B}$ at $0-16 \mathrm{~min}, 11-16 \% \mathrm{~B}$ at $16-28 \mathrm{~min}, 16 \% \mathrm{~B}$ at $28-50 \mathrm{~min}, 16$ $30 \% \mathrm{~B}$ at $50-65 \mathrm{~min}, 30-90 \% \mathrm{~B}$ at $65-70 \mathrm{~min}, 90 \% \mathrm{~B}$ at $70-75 \mathrm{~min}$, $90-11 \% \mathrm{~B}$ at $75-80 \mathrm{~min}, 11 \% \mathrm{~B}$ at $80-85 \mathrm{~min}$. The flow rate was $1.0 \mathrm{ml} / \mathrm{min}$ and aliquots of $15 \mu \mathrm{l}$ were injected.

HPLC-MS System and Conditions Mass spectrometry was used to confirm the chromatographic profile obtained by DAD detection. The MS analysis was performed on an Agilent G1969LC/MSD TOF system (Agilent Corp. Santa Clara, CA, U.S.A.) equipped with an ESI interface. The TOFMS analysis worked in negative mode using full scan mode and mass range was set at $100-3000 \mathrm{Da}$. The conditions of ESI source were as follows: drying gas $\left(\mathrm{N}_{2}\right)$ flow rate, $10.0 \mathrm{l} / \mathrm{min}$; drying gas temperature, $320^{\circ} \mathrm{C}$; nebulizer, $241 \mathrm{kPa}(35 \mathrm{psig}$ ); capillary voltage, $4000 \mathrm{~V}$; fragmentor $120 \mathrm{~V}$; skimmer voltage, $60 \mathrm{~V}$; octapole DC1, $37 \mathrm{~V}$; octapole RF, $250 \mathrm{~V}$. Data were acquired and analyzed by LC-MS TOF Software Ver. A.01.00 (Agilent Technologies) and PE Sciex Analyst QS 1.1

Sample Preparation The samples were powdered to a homogeneous size in a mill, passed though a 40 -mesh sieve, and dried at $60^{\circ} \mathrm{C}$ in the oven for $3 \mathrm{~h}$. The accurately weighed powder $(1.0 \mathrm{~g})$ was suspended in $30 \mathrm{ml} 50 \%$ methanol (v/v), ultrasonically extracted for $45 \mathrm{~min}$, and then cooled at room temperature; $50 \%$ methanol was added to compensate for the lost weight. The methanol solution was filtered through a $0.45 \mu \mathrm{m}$ membrane prior to HPLC.

Calibration Curves, Limits of Detection and Quantification A methanol stock solution, which contained thirteen analytes, was prepared and diluted to an appropriate concentration for the construction of calibration curves. Six concentrations of the mixed standard solution were injected in triplicate. The calibration curves were constructed by plotting the mean peak areas versus the concentration of standards. The lowest concentration of working solution was diluted with methanol to yield a series of appropriate concentrations, and the limit of detection (LOD) and quantification (LOQ) under the chromatographic conditions were separately determined at a signal-to-noise ratio $(\mathrm{S} / \mathrm{N})$ of 3 and 10 , respectively.

Precision, Accuracy and Stability The measurement of intra-day and inter-day variability was utilized to determine the precision of this newly developed method. The intra-day variation was determined by analyzing in triplicate the same mixed standard methanol solution for three times within $1 \mathrm{~d}$. While for inter-day variability test, the solution was examined in triplicate for consecutive $3 \mathrm{~d}$. The relative standard deviation (RSD) was taken as a measure of precision. Recovery test was used to evaluate the accuracy of the developed assay. Accurate amounts of the thirteen standards were added to approximate $0.5 \mathrm{~g}$ of Flos Lonicerae powder (sample 1), and then extracted and analyzed as described above. Each sample was analyzed in triplicate. The average percentage recoveries were evaluated by calculating the ratio of detected amount versus added amount. Stability of sample solution was tested at $4{ }^{\circ} \mathrm{C}$. The sample solution was analyzed in triplicate every $8 \mathrm{~h}$ within $48 \mathrm{~h}$. The analytes showed very stable in methanol solution $(\mathrm{RSD}<3.5 \%)$ over the tested peroid.
Table 1. Calibration Curves, LODs and LOQs of Thirteen Analytes

\begin{tabular}{cccccc}
\hline \hline Analyte & $\begin{array}{c}\text { Calibration } \\
\text { curve }\end{array}$ & $r^{2}$ & $\begin{array}{c}\text { Test range } \\
(\mu \mathrm{g} / \mathrm{ml})\end{array}$ & $\begin{array}{c}\mathrm{LOD}^{b)} \\
(\mu \mathrm{g} / \mathrm{ml})\end{array}$ & $\begin{array}{c}\mathrm{LOQ}^{c)} \\
(\mu \mathrm{g} / \mathrm{ml})\end{array}$ \\
\hline $\mathbf{1}$ & $y=3683.2 x+9.82$ & 0.9997 & $21-1040$ & 0.11 & 0.61 \\
$\mathbf{2}$ & $y=3800.3 x+6.83$ & 0.9993 & $1.1-44$ & 0.12 & 0.82 \\
$\mathbf{3}$ & $y=2033.7 x-1.56$ & 0.9987 & $1.2-48$ & 0.13 & 0.85 \\
$\mathbf{4}$ & $y=1244.2 x-4.41$ & 0.9994 & $9.0-360$ & 0.18 & 1.21 \\
$\mathbf{5}$ & $y=1462.8 x-2.18$ & 0.9997 & $10.5-420$ & 0.15 & 1.10 \\
$\mathbf{6}$ & $y=1489.7 x-2.41$ & 0.9994 & $2.5-100$ & 0.14 & 0.93 \\
$\mathbf{7}$ & $y=2712.3 x-0.61$ & 0.9999 & $4.7-188$ & 0.12 & 0.75 \\
$\mathbf{8}$ & $y=3824.1 x-6.94$ & 0.9999 & $4.3-172$ & 0.13 & 0.64 \\
$\mathbf{9}$ & $y=1643.2 x-7.12$ & 0.9983 & $3.0-120$ & 0.15 & 0.91 \\
$\mathbf{1 0}$ & $y=2159.1 x-4.22$ & 0.9984 & $3.8-144$ & 0.14 & 0.83 \\
$\mathbf{1 1}$ & $y=3740.4 x+3.56$ & 0.9986 & $15.8-630$ & 0.10 & 0.77 \\
$\mathbf{1 2}$ & $y=2912.1 x+1.32$ & 0.9997 & $15.5-620$ & 0.14 & 0.91 \\
$\mathbf{1 3}$ & $y=1904.7 x-2.15$ & 0.9999 & $21.0-840$ & 0.18 & 1.13 \\
\hline
\end{tabular}

a) $y$ : peak area; $x$ : concentration of the analyte $(\mu \mathrm{g} / \mathrm{ml}) . \quad$ b) LOD, limit of detection. c) LOQ, limit of quantification.

\section{Results and Discussion}

Optimization of Chromatographic Conditions Chromatographic conditions were optimized. Various compositions of mobile phase were tried. As a result, acetonitrile and $0.4 \%$ acetic acid aqueous was chosen as the eluting solvent system to give the desired separation and acceptable tailing factor within the running time of $60 \mathrm{~min}$. As the three types of compounds show different UV absorption properties, different detection wavelengths were simultaneously set to monitor these compounds in a single run, viz. $240 \mathrm{~nm}$ for iridoids 3, 4, 5 and 13, $330 \mathrm{~nm}$ for phenolic acids 1, 2, 11 and 12, $360 \mathrm{~nm}$ for flavonoids $6,7, \mathbf{8}, 9$ and $\mathbf{1 0}$. The detection parameters provided sufficient sensitivity for each analytes (Table 1). Representative chromatograms of mixed standards and Flos Lonicerae were shown in Fig. 2 and 3, respectively.

Validation of the Method All calibration curves showed good linear regression $\left(r^{2}>0.9983\right)$ within test ranges. The overall intra-day and inter-day variations were less than $2.4 \%$ for all analytes. As shown in Table 2, the developed analytical method provided good accuracy with the recoveries from 91.3 to $104.2 \%$ for the analytes. The limit of detection $(\mathrm{S} / \mathrm{N}=3)$ and the limit of quantification $(\mathrm{S} / \mathrm{N}=10)$ were less than $0.19 \mu \mathrm{g} / \mathrm{ml}$ and $1.22 \mu \mathrm{g} / \mathrm{ml}$, respectively. Therefore, this verified HPLC-DAD method was precise, accurate and sensitive for the quantitative evaluation of major bioactive compounds in Flos Lonicerae.

Peak Identifications Considering that the chromatographic peaks could not be identified unambiguously only by retention time and UV spectra in a considerable complex matrix, HPLC-MS was used as a necessary supplement for confirmation of peak identification. Thus, peaks were identified by three means in this study: (1) by comparing the retention times of the unknown peaks with reference compounds, (2) by comparing the UV data with reference compounds, and (3) by comparing the MS data with reference compounds. The total ion current chromatogram (TIC) of the reference compounds and the Flos Lonicerae sample were shown in Fig. 4, the data of peak identifications were list in Table 3 .

Sample Analysis This newly proposed HPLC-DAD-MS method was subsequently applied to the simultaneous determination of thirteen bioactive components in ten commercial Flos Lonicerae samples obtained from local markets all 


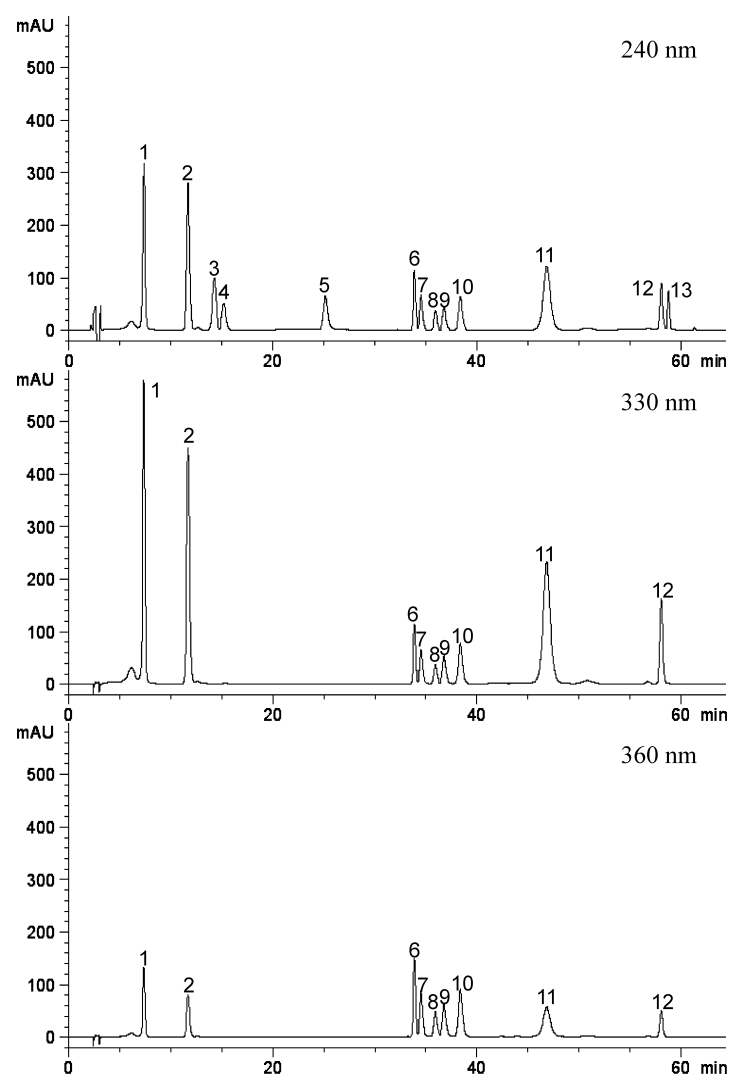

Fig. 2. Typical HPLC Chromatograms of Reference Compounds (A) $240 \mathrm{~nm}$ for the 13 compounds; (B) $330 \mathrm{~nm}$ for the 13 compounds; (C) $360 \mathrm{~nm}$ for the 13 compounds.

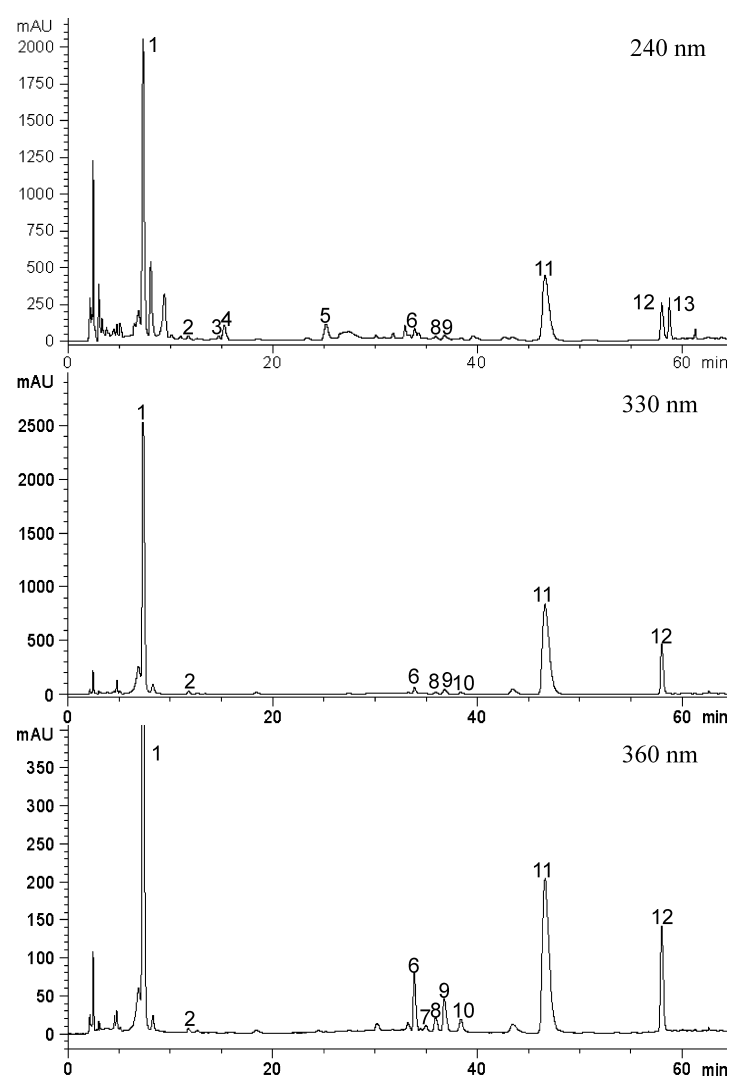

Fig. 3. Typical HPLC Chromatograms of Flos Lonicerae Sample

(A) $240 \mathrm{~nm}$ for the Flos Lonicerae; (B) $330 \mathrm{~nm}$ for the Flos Lonicerae; (C) $360 \mathrm{~nm}$ for Flos Lonicerae.
Table 2. Precisions and Recoveries of the Thirteen Analytes

\begin{tabular}{cccccc}
\hline \hline & \multicolumn{2}{c}{ Precision } & & \multicolumn{2}{c}{ Recovery $(n=3)$} \\
\cline { 2 - 3 } \cline { 5 - 6 } Analyte & $\begin{array}{c}\text { Intra-day } \\
(n=9) \\
\text { RSD }(\%)\end{array}$ & $\begin{array}{c}\text { Inter-day } \\
(n=9) \\
\text { RSD }(\%)\end{array}$ & & $\begin{array}{c}\text { Recovery } \\
(\%)\end{array}$ & $\begin{array}{c}\text { RSD } \\
(\%)\end{array}$ \\
& 1.31 & 1.65 & & 95.2 & 1.84 \\
$\mathbf{1}$ & 1.19 & 1.52 & & 103.6 & 2.84 \\
$\mathbf{2}$ & 1.85 & 2.12 & & 96.3 & 2.36 \\
$\mathbf{3}$ & 1.24 & 1.93 & & 104.2 & 1.65 \\
$\mathbf{4}$ & 1.32 & 1.41 & & 98.2 & 1.76 \\
$\mathbf{5}$ & 1.21 & 1.86 & & 91.3 & 1.96 \\
$\mathbf{6}$ & 1.35 & 1.57 & & 97.8 & 2.82 \\
$\mathbf{7}$ & 1.10 & 2.36 & & 96.6 & 1.56 \\
$\mathbf{8}$ & 0.93 & 1.96 & & 101.2 & 2.76 \\
$\mathbf{9}$ & 1.36 & 1.82 & & 92.4 & 2.11 \\
$\mathbf{1 0}$ & 0.81 & 1.46 & & 103.2 & 0.83 \\
$\mathbf{1 1}$ & 0.78 & 1.13 & & 94.4 & 1.93 \\
$\mathbf{1 2}$ & 1.73 & 2.26 & & 95.6 & 1.21 \\
$\mathbf{1 3}$ & & & &
\end{tabular}
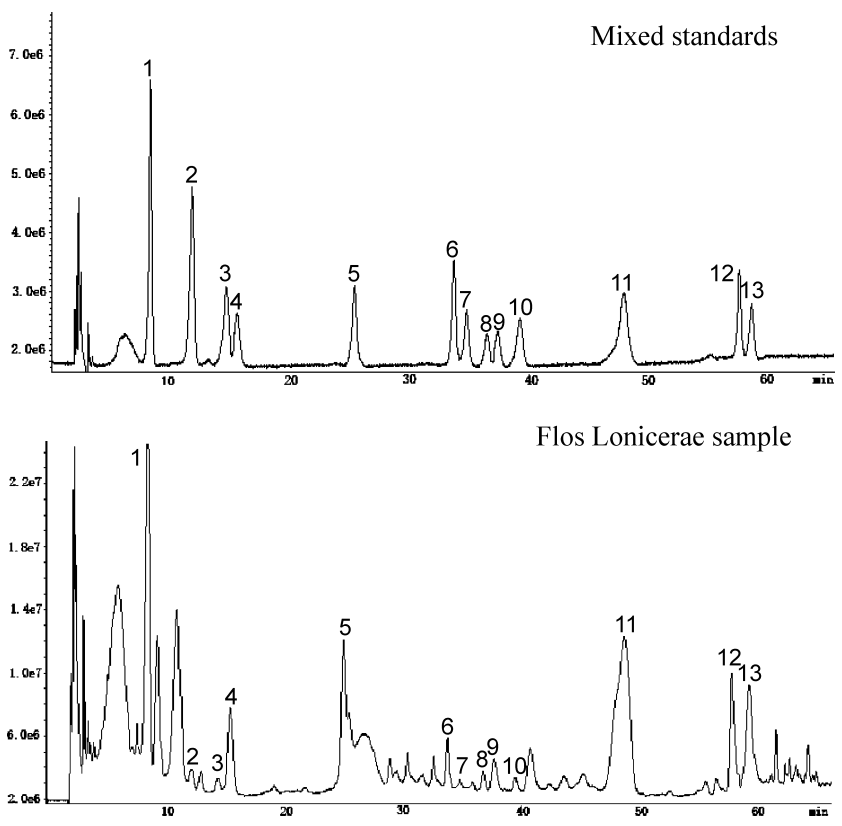

Fig. 4. TIC of Reference Compounds and Flos Lonicerae Sample

around China. Their contents were summarized in Table 4. The result indicated that phenolic acids were present in the highest concentrations, followed by iridoids. The flavonoids were present in the lowest concentrations. For each of the compounds, there was a relatively large range of concentrations among the tested samples, suggesting the inconsistent quality of commercial Flos Lonicerae. The analytical results obtained also indicated that a multiple-component-assay including phenolic acids, iridoids and flavonoids might be a rational strategy to elucidate the synergic effects and comprehensively control the quality of this herbal drug.

\section{Conclusions}

In this study, an HPLC-DAD-MS method for the qualification and quantification of three groups of compounds (phenolic acids, iridoids and flavonoids) in Flos Lonicerae has been developed and successfully applied to ten commercial Flos Lonicerae samples. This method is validated for good 
Table 3. HPLC-DAD-MS Data of the Thirteen Bioactive Compounds in Reference Solution and Flos Lonicerae Sample

\begin{tabular}{|c|c|c|c|c|c|c|c|}
\hline \multirow[b]{2}{*}{ Peak } & \multicolumn{3}{|c|}{ Reference solution } & \multicolumn{3}{|c|}{ Flos Lonicerae sample solution } & \multirow[b]{2}{*}{ Compound identity } \\
\hline & $\begin{array}{c}t_{\mathrm{R}}^{a)} \\
(\mathrm{min})\end{array}$ & $\begin{array}{l}\lambda_{\max } \\
(\mathrm{nm})\end{array}$ & $\begin{array}{c}{[\mathrm{M}-\mathrm{H}]^{-}} \\
\quad(\mathrm{m} / \mathrm{z})\end{array}$ & $\begin{array}{c}t_{\mathrm{R}}^{a)} \\
(\mathrm{min})\end{array}$ & $\begin{array}{l}\lambda_{\max } \\
(\mathrm{nm})\end{array}$ & $\begin{array}{c}{[\mathrm{M}-\mathrm{H}]^{-}} \\
\quad(\mathrm{m} / \mathrm{z})\end{array}$ & \\
\hline 1 & 7.4 & 330 & 353 & 7.3 & 330 & 353 & Chlorogenic acid \\
\hline 2 & 12.1 & 330 & 179 & 11.9 & 330 & 179 & Caffeic acid \\
\hline 3 & 14.4 & 240 & 389 & 14.2 & 240 & 389 & Loganin \\
\hline 4 & 15.6 & 250 & 357 & 15.4 & 250 & 357 & Sweroside \\
\hline 5 & 25.7 & 235 & 403 & 25.5 & 235 & 403 & Secoxyloganin \\
\hline 6 & 33.8 & 255,360 & 609 & 33.6 & 255,360 & 609 & Rutin \\
\hline 7 & 34.5 & 255,360 & 463 & 34.2 & 255,360 & 463 & Hyperoside \\
\hline 8 & 36.4 & 255,360 & 463 & 36.2 & 255,360 & 463 & Quercetin-3-O-glucoside \\
\hline 9 & 36.9 & 260,355 & 447 & 36.7 & 260,355 & 447 & Luteolin-7-O-glucoside \\
\hline 10 & 38.7 & 260,355 & 593 & 38.5 & 260,355 & 593 & Lonicerin \\
\hline 11 & 47.7 & 330 & 515 & 47.6 & 330 & 515 & 4,5-Di-O-caffeoyl quinic acid \\
\hline 12 & 57.8 & 330 & 515 & 57.6 & 330 & 515 & 3,4-Di- $O$-caffeoyl quinic acid \\
\hline 13 & 58.8 & 240 & 757 & 58.7 & 240 & 757 & Centauroside \\
\hline
\end{tabular}

a) $t_{\mathrm{R}}$ : retention time.

Table 4. Content of Thirteen Compounds in Samples of Flos Lonicerae

\begin{tabular}{|c|c|c|c|c|c|c|c|c|c|c|c|c|c|}
\hline \multirow{2}{*}{ Sample } & \multicolumn{13}{|c|}{ Content (mg/g) } \\
\hline & 1 & 2 & 3 & 4 & 5 & 6 & 7 & 8 & 9 & 10 & 11 & 12 & 13 \\
\hline 1 & 16.973 & 0.172 & 0.088 & 2.420 & 2.685 & 0.871 & $\mathrm{nd}^{b)}$ & 0.131 & 0.640 & 0.192 & 10.525 & 3.401 & 2.182 \\
\hline 2 & 17.144 & 0.195 & 0.270 & 3.749 & 2.677 & 1.120 & nd & 0.196 & 0.647 & 0.276 & 14.123 & 4.319 & 2.870 \\
\hline 3 & 15.263 & 0.295 & $\operatorname{tr}^{a)}$ & 3.173 & 2.572 & 0.829 & nd & 0.127 & 0.554 & 0.237 & 12.091 & 5.624 & 2.177 \\
\hline 4 & 17.531 & 0.512 & 0.101 & 2.448 & 2.469 & 0.775 & nd & 0.331 & 0.623 & 0.188 & 11.637 & 4.177 & 1.967 \\
\hline 5 & 18.057 & 0.203 & 0.783 & 2.349 & 3.832 & 1.001 & nd & 0.217 & 0.541 & 0.219 & 12.551 & 2.249 & 1.267 \\
\hline 6 & 16.876 & 0.359 & 0.109 & 3.853 & 2.483 & 1.034 & $\operatorname{tr}$ & 0.189 & 0.551 & 0.269 & 12.067 & 5.445 & 2.319 \\
\hline 7 & 16.946 & 0.475 & 0.054 & 0.699 & 1.305 & 0.670 & nd & 0.106 & 0.460 & 0.164 & 9.763 & 2.116 & 1.094 \\
\hline 8 & 15.097 & 0.283 & 0.134 & 3.887 & 2.310 & 1.005 & $\operatorname{tr}$ & 0.172 & 0.477 & 0.321 & 11.696 & 4.613 & 2.379 \\
\hline 9 & 16.505 & 0.342 & 0.080 & 3.834 & 2.084 & 0.939 & nd & 0.175 & 0.485 & 0.234 & 12.122 & 5.173 & 2.239 \\
\hline 10 & 16.191 & 0.286 & 0.084 & 3.636 & 2.676 & 1.027 & nd & 0.173 & 0.515 & 0.276 & 12.092 & 4.709 & 2.722 \\
\hline
\end{tabular}

a) tr: trace. b) nd: not detected.

accuracy, repeatability and precision, and can be used as a valid analytical method to evaluate the intrinsic quality of Flos Lonicerae.

Acknowledgements This research was financially supported by Distinguished Young Scholars from National Science Foundation of China (No. 30325046)

\section{References}

1) "Pharmacopoeia of the People's Republic of China," Chemical Industry Press, Peking, 2005, p. 152.

2) Ge B., Lu X. Y., Yi K., Tian Y., Chin. Wild Plant Resour., 23, 13-16 (2004).

3) Zhao G. L., Liu J. J., Dan D., Zhang X. H., Wang H., Chin. Tradit. Pat. Med., 24, 973-976 (2002).

4) Chang C. W., Lin M. T., Lee S. S., Karin C. S., Liu C., Hsu F. L., Lin J. Y., Antivir. Res., 27, 367-374 (1995).

5) Chang W. C., Hsu F. L., Prostag. Leukotr. Ess., 45, 307-312 (1992).

6) Sun Y. B., Wang Y., Guan X. Z., Feng Y. M., Zhao Y. C., Chin. J. Chin. Mater. Med., 21, 242-243 (1996).

7) Xiang T., Xiong Q. B., Ketut A. I., Tezuka Y., Nagaoka T., Wu L. J., Kadota S., Planta Med., 67, 323-325 (2001).

8) Shi Y., Shi R. B., Lu W. R., Chin. Pharm. J., 34, 724-727 (1999).

9) Leung H. W. C., Kuo C. L., Yang W. H., Lin C. H., Lee H. Z., Eur. J.
Pharmacol., 534, 12-18 (2006).

10) Lee S. J., Choi J. H., Chang H. W., Kang S. S., Kim H. P., Life Sci., 57, $551-558$ (1995).

11) Xing J. B., Li P., Chin. Med. Mater, 22, 366-370 (1999).

12) Chen J., Li S. L., Li P., Song Y., Chai X. Y., Ma D. Y., J. Sep. Sci., 28, 365-372 (2005)

13) Wang T. Z., Li Y. M., Wang Z. X., Chin. J. Pharm. Anal., 20, 293296 (2000).

14) Li H. J., Li P., Chem. Ind. Prod., 25, 29-32 (2005).

15) Mathad V. T., Raj K., Bhaduri A. P., Sahai R., Puri A., Tripathi L. M., Srivastava V. M. L., Bioorg. Med. Chem., 6, 605-611 (1998).

16) Kumarasamy Y., Nahar L., Cox P. J., Jaspars M., Sarker S. D., Phytomedicine, 10, 344-347 (2003).

17) Li H. J., Li P., Ye W. C., J. Chromatogr. A, 1008, 167-172 (2003).

18) Song Y., Li S. L., Wu M. H., Li H. J., Li P., Anal. Chim. Acta, 564, $211-218(2006)$

19) Xing J. B., Li H. J., Li P., Liu Y., Chin. J. New Drugs, 11, 856-896 (2002).

20) Chai X. Y., Li P., Tang L. Y., Chin. J. Chin. Mater. Med., 35, 865-867 (2004).

21) Gao Y. M., Wang M. Z., Wang J. P., Zhan Q., Qin H. Y., Mu H. J., Guan G. J., Wang H., Chin. Tradit. Herb. Drugs, 26, 568-569 (1995).

22) Chang C. W., Lin M. T., Lee S. S., Liu K. C., Hsu F. L., Lin J. Y., Antivir. Res., 27, 367-374 (1995).

23) Li H. J., Li P., Chin. Pharm. J., 41, 818-819 (2006). 